Mathematical Research Letters 1, 1-9 (1994)

\title{
A REMARK ON TUBE DOMAINS
}

\author{
Benedict H. Gross
}

Let $D$ be a tube domain (i.e. a bounded symmetric domain of tube type). In $[D, \S 1]$, Deligne gives a description of $D$ as the moduli space of certain Hodge structures. Using these methods, we show that $D$ parametrizes a canonical variation $\mathcal{V}$ of polarized real Hodge structures, which is effective of weight $=\operatorname{rank}(D)$ and enjoys several remarkable properties. We end with some speculation on how $\mathcal{V}$ might appear in algebraic geometry.

1.

Let $D$ be a simple tube domain, and let $G$ be (the real points of) the simply-connected, simple real algebraic group which acts transitively on $D$. Let $K$ be a maximal compact subgroup of $G$; then $K$ fixes a unique point of $D$ and $D \simeq G / K$. The integer $n=\operatorname{rank}(D)$ is defined to be the real rank of $G$, and the integer $d=\operatorname{dim}(D)$ is defined to be the complex dimension of the domain, which is one-half the real dimension of $G / K$. The quotient $2 d / n$ is always an integer [S, p. 37].

Since $D$ is tube, there is a self-adjoint homogeneous cone $C$ in a Euclidean space $N$ over $\mathbb{R}$ such that $D \simeq N+i C \subseteq N_{\mathbb{C}}[\mathrm{S}$, p. 128].

We recall $[\mathrm{D}, 1.2 .6]$ that the simple bounded symmetric domains are classified by pairs $(\Delta, v)$, where $\Delta$ is a connected Dynkin diagram and $v$ is a special vertex of $\Delta$ which is equivalent to the extended vertex $\mu$ under an automorphism of the affine diagram $\Delta^{\prime}=\Delta \cup\{\mu\}$ [T, pp. 33-34, p. 53]. The domain is tube if $v$ is fixed by the opposition involution of $\Delta$.

We now tabulate the relevant pairs $(\Delta, v)$, where the special vertex is circled, and give the groups $G$ and $K$ associated to the tube domain $D$. We also describe the cone $C$, using the notation $S_{n}(F)^{+}$for the cone of positive definite, $n \times n$ Hermitian symmetric matrices over the $\mathbb{R}$-algebras $F=\mathbb{R}, \mathbb{C}, \mathbb{H}$.

Received August 11, 1993. 


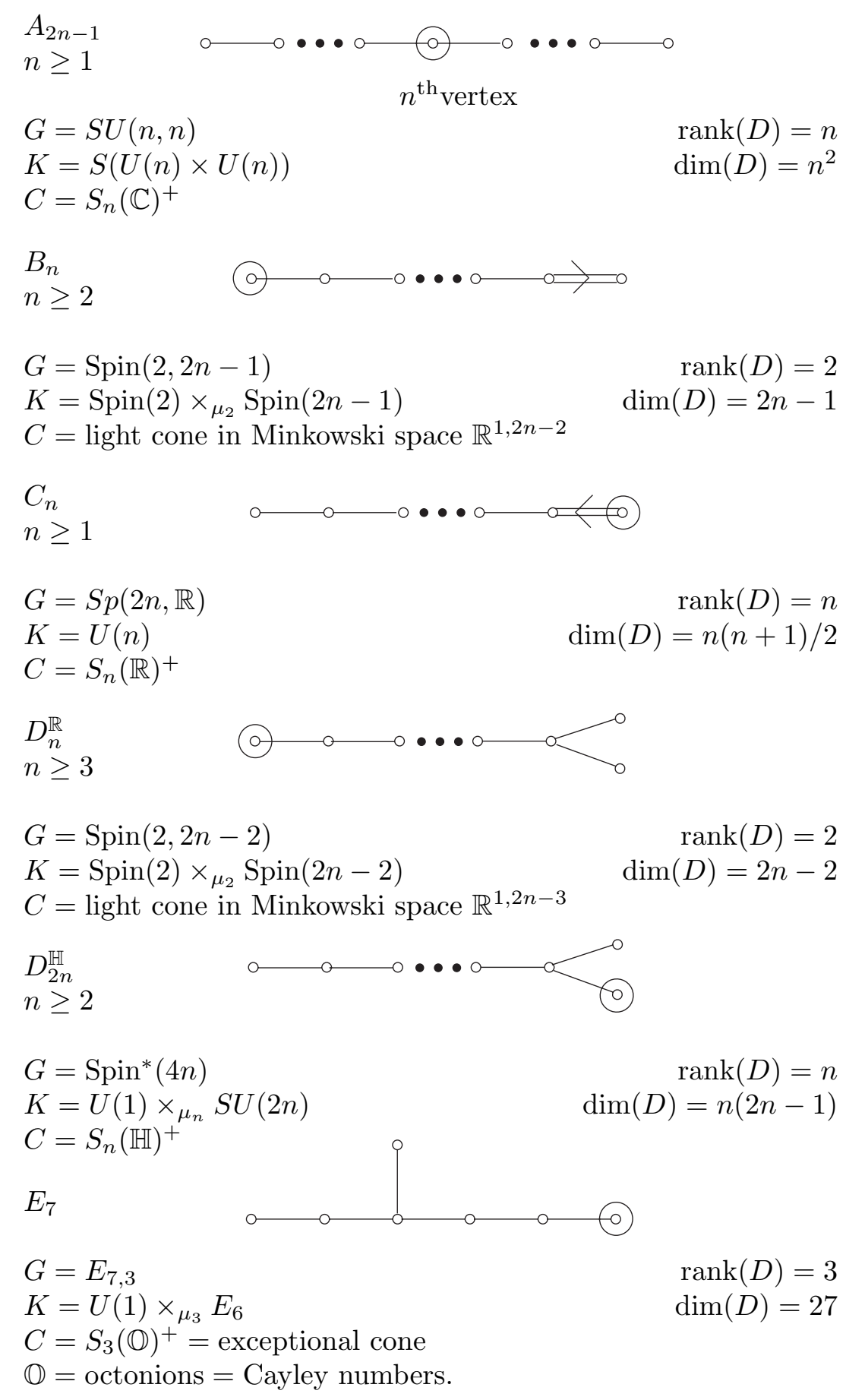


2.

The vertex $v$ of $\Delta$ determines a conjugacy class of maximal parabolic subgroups $P=L \cdot N$ in $G$, with unipotent radical $N$ abelian and Levi subgroup $L$ a real form of $K$. The cone $C$ is $L$-homogeneous in the real vector space $N$.

The vertex $v$ also determines a fundamental, irreducible representation $V$ of $G$ over $\mathbb{R}$. The orbit of a highest weight vector in $\mathbb{P}(V)$ is the real projective variety $G / P$. We describe the fundamental representation $V$, in terms of the standard representation of $G$, in the table below.

$\begin{array}{cccc}\text { Type } & G & V & \operatorname{dim} V \\ A & S U(n, n) & V_{\mathbb{C}}=\bigwedge^{n} \mathbb{C}^{2 n} & \left(\begin{array}{c}2 n \\ n\end{array}\right) \\ B, D^{R} & \operatorname{Spin}(2, m) & \mathbb{R}^{2, m} \text { of } S O(2, m) & 2+m \\ C & S p(2 n, \mathbb{R}) & V \oplus \bigwedge^{n-2} \mathbb{R}^{2 n}=\bigwedge^{n} \mathbb{R}^{2 n} & \left(\begin{array}{c}2 n \\ n\end{array}\right)-\left(\begin{array}{c}2 n \\ n-2\end{array}\right) \\ D^{H} & \operatorname{Spin}^{*}(4 n) & \text { unique } \frac{1}{2} \text {-spin which is real } & 2^{2 n-1} \\ E & E_{7,3} & \text { unique miniscule representation } & 56\end{array}$

3.

We will see that the fundamental representation $V$ gives rise to a canonical variation of polarized real Hodge structures on $D$. To do this, following Deligne $[\mathrm{D}, \S 1]$, we must first realize $V$ as a representation of a reductive group $G_{1}$, whose center contains $\mathbb{G}_{m}$ and whose derived group is $G$. We construct $G_{1}$ as follows.

Let $\epsilon$ be the unique element of order 2 in the center of $G$ which is contained in the connected component of the center of $K$. We recall that $Z(K)^{+} \simeq U(1)$ in all cases. Let $G_{1}$ be the quotient of $\mathbb{G}_{m} \times G$ by the central subgroup generated by the involution $-1 \times \epsilon$. Since $\epsilon$ acts as $(-1)^{n}$ on $V$, with $n=\operatorname{rank}(D), V$ extends uniquely to a representation of $G_{1}$ such that $\lambda \in \mathbb{G}_{m}$ acts by $\lambda^{-n}$.

Let $S=\operatorname{Res}_{\mathbb{C} / \mathbb{R}} \mathbb{G}_{m}=\mathbb{G}_{m} \times U(1) /\langle-1 \times-1\rangle$. A point of $D$ determines a homomorphism $U(1) \hookrightarrow K \hookrightarrow G$, which is an oriented isomorphism from $U(1)$ to the connected component of the center of $K$. Since this maps the element -1 of $U(1)$ to the element $\epsilon$ of $G$, it determines a homomorphism

$$
h: S \longrightarrow G_{1}
$$

which is the identity on $\mathbb{G}_{m}$. The $G_{1}$-conjugacy class $X$ of $h$ has two connected components, each of which is isomorphic to $D$.

Finally, the representation $V$ of $G_{1}$, when combined with the morphism $h$ of (3.1), gives rise to a polarized variation of real Hodge structures on 
$X$ (and hence on $D$ ), by the results of Deligne [D, Prop. 1.1.14], [M, Ch. II, Prop. 3.2]. We put $\mathcal{V}=V \otimes \mathcal{O}_{D}$; this is an equivariant holomorphic vector bundle on $D$ with connection $\nabla$. Since $\lambda \in \mathbb{G}_{m}$ acts as $\lambda^{-n}$ on $V$, the Hodge structures associated to $V$ are pure of weight $n=\operatorname{rank}(D)$.

4.

We now investigate the properties of $\mathcal{V}$ as a variation of Hodge structure. We recall that $\mathcal{V}$ has a filtration by holomorphic sub-bundles $\cdots \supset F^{p} \mathcal{V} \supset$ $F^{p+1} \mathcal{V} \supset \cdots$, and that the quotient bundles

$$
\mathcal{W}^{p, q}=F^{p} \mathcal{V} / F^{p+1} \mathcal{V} \quad p+q=n
$$

are equivariant vector bundles on $X$. On $D \simeq G / K$, these bundles correspond to the $K$-submodules $W^{p, q}$ of $V_{\mathbb{C}}$ on which elements $z \in U(1)=$ $Z(K)^{+}$act by the character $z^{-p} \bar{z}^{-q}=z^{q-p}$.

Proposition 4.1. The variation of Hodge structures $\mathcal{V}$ is effective of weight $n$, so $\mathcal{W}^{p, q}=0$ unless both $p, q \geq 0$.

If $p, q \geq 0$ the equivariant vector bundle $\mathcal{W}^{p, q}$ is irreducible. Both $F^{n} \mathcal{V}=$ $W^{n, 0}$ and $\mathcal{V} / F^{1} \mathcal{V}=\mathcal{W}^{0, n}$ are holomorphic line bundles on $X$.

Proof. This results from a determination of the eigenspaces $W^{p, q}$ for the action of $U(1) \simeq Z(K)^{+}$on $V_{\mathbb{C}}$, as representations of $K$. Only the characters $z^{n}, z^{n-2}, \cdots, z^{2-n}, z^{-n}$ occur, and we tabulate these representations below.

$\begin{array}{cclc}\text { Type } & K & W^{p, q} \quad p, q \geq 0 & \operatorname{dim} W^{p, q} \\ A & S(U(n) \times U(n)) & \left(\bigwedge^{p} \mathbb{C}^{n}\right)^{*} \otimes\left(\bigwedge^{q} \mathbb{C}^{n}\right)^{*} & \left(\begin{array}{c}n \\ p\end{array}\right)\left(\begin{array}{l}n \\ q\end{array}\right) \\ B, D^{\mathbb{R}} & \operatorname{Spin}(2) \times_{\mu_{2}} \operatorname{Spin}(m) & W^{2,0}=\mathbb{C}(-2) \otimes \mathbb{C} & 1 \\ & & W^{1,1}=\mathbb{C} \otimes \mathbb{C}^{m} & m \\ & & W^{0,2}=\mathbb{C}(2) \otimes \mathbb{C} & 1 \\ C & U(n) & \text { Irreducible summand of } & \left(\begin{array}{c}n \\ p\end{array}\right)\left(\begin{array}{c}n \\ q\end{array}\right)-\left(\begin{array}{c}n \\ p-1\end{array}\right)\left(\begin{array}{c}n \\ q-1\end{array}\right) \\ & & \left(\bigwedge^{p} \mathbb{C}^{n}\right)^{*} \otimes \bigwedge^{q} \mathbb{C}^{n} \text { with } & \\ & & \text { highest weight }\left(=2 \omega_{q}\right) & \\ D^{\mathbb{H}} & U(1) \times_{\mu_{n}} S U(2 n) & \mathbb{C}(q-p) \otimes\left(\bigwedge^{2 p} \mathbb{C}^{2 n}\right)^{*} & \left(\begin{array}{l}2 n \\ 2 p\end{array}\right) \\ E & U(1) \times_{\mu_{3}} E_{6} & W^{3,0}=\mathbb{C}(-3) \otimes \mathbb{C} & 1 \\ & & W^{2,1}=\mathbb{C}(-1) \otimes W_{27} & 27 \\ & & W^{1,2}=\mathbb{C}(1) \otimes W_{27}^{*} & 27 \\ & & W^{0,3}=\mathbb{C}(3) \otimes \mathbb{C} & 1\end{array}$


5.

The connection $\nabla: \mathcal{V} \rightarrow \mathcal{V} \otimes \Omega_{X}^{1}$ satisfies Griffiths transversality: $\nabla\left(F^{p} \mathcal{V}\right) \subset F^{p-1} \mathcal{V} \otimes \Omega_{X}^{1}$. Hence, if $\Theta_{X}$ is the holomorphic tangent bundle of $X$, differentiating $q$ times gives a morphism of equivariant vector bundles

$$
\nabla^{q}: \operatorname{Sym}^{q} \Theta_{X} \longrightarrow \operatorname{Hom}\left(F^{n} \mathcal{V}, F^{p} \mathcal{V} / F^{p+1} \mathcal{V}\right)
$$

where $p+q=n$.

Proposition 5.2. The morphism $\nabla^{q}$ is surjective for all $0 \leq q \leq n$, and

$$
\nabla: \Theta_{X} \longrightarrow \operatorname{Hom}\left(F^{n} \mathcal{V}, F^{n-1} \mathcal{V} / F^{n} \mathcal{V}\right)
$$

is an isomorphism.

Proof. Since $\operatorname{Hom}\left(F^{n} \mathcal{V}, F^{p} \mathcal{V} / F^{p+1} \mathcal{V}\right) \simeq \mathcal{W}^{p, q} \otimes\left(F^{n} \mathcal{V}\right)^{-1}$ is an irreducible equivariant bundle, it suffices to check that $\nabla^{q} \neq 0$. This reduces to the study of the action of elements in $N_{\mathbb{C}}^{-} \subset G_{\mathbb{C}}$ on the eigenspace $W^{n, 0}$ of $V_{\mathbb{C}}$ (cf. [D, Prop. 1.1.14]). We leave the details to the reader. Since $\operatorname{dim}\left(W^{n-1,1}\right)=\operatorname{dim}(X)=d$ and the map $\nabla$ is surjective, it is an isomorphism.

The maps $\nabla^{q}$ defined in (5.1) give a surjection $f=\bigoplus_{q \geq 0} \nabla^{q}$ of vector bundles on $X$ :

$$
\operatorname{Sym}^{\bullet}\left(\Theta_{X}\right)=\bigoplus_{q \geq 0} \operatorname{Sym}^{q} \Theta_{X} \stackrel{f}{\longrightarrow} \bigoplus_{q=0}^{n} \mathcal{W}^{p, q} \otimes\left(\mathcal{W}^{n, 0}\right)^{-1} \longrightarrow 0 .
$$

The kernel of $f$ is a graded ideal $I=\bigoplus_{q \geq 2} I^{q}$ of $\operatorname{Sym} \bullet\left(\Theta_{X}\right)$, probably generated by the irreducible equivariant bundle $I^{2}=\operatorname{ker}\left(\nabla^{2}: \operatorname{Sym}^{2} \Theta_{X} \longrightarrow\right.$ $\left.\mathcal{W}^{n-2,2} \otimes\left(\mathcal{W}^{n, 0}\right)^{-1}\right)$.

6.

If $D$ is any tube domain, it admits a product decomposition $D=$ $D_{1} \times D_{2} \times \cdots \times D_{k}$ into simple tube domains of the type classified in $\S 1$. The variation $\mathcal{V}=\mathcal{V}_{1} \otimes \mathcal{V}_{2} \otimes \cdots \otimes \mathcal{V}_{k}$ of polarized real Hodge structures on $D$ is effective of weight $n=\operatorname{rank}(D)=\sum_{i=1}^{k} n_{i}, F^{n} \mathcal{V}=F^{n_{1}} \mathcal{V}_{1} \otimes$ $F^{n_{2}} \mathcal{V}_{2} \otimes \cdots \otimes F^{n_{k}} \mathcal{V}_{k}$ is a holomorphic line bundle on $D, \nabla^{q}: \operatorname{Sym}^{q} \Theta_{D} \longrightarrow$ $\operatorname{Hom}\left(F^{n} \mathcal{V}, F^{p} \mathcal{V} / F^{p+1} \mathcal{V}\right)$ is surjective for all $0 \leq q \leq n$, and $\nabla=\nabla^{1}$ is an isomorphism. These properties characterize $\mathcal{V}$ on $D$.

We end with some remarks on the irreducible representation $V=V_{1} \otimes$ $V_{2} \otimes \cdots \otimes V_{k}$ of $G=G_{1} \times G_{2} \times \cdots \times G_{k}$ for the general tube domain $D$. Let 
$n$ be the real rank of $G$, and let $\left\{\gamma_{1}, \gamma_{2}, \cdots, \gamma_{n}\right\}$ be a system of strongly orthogonal, non-compact positive roots for a compact torus $T$ in $G[\mathrm{~S}$, p. 60]. Then

$$
\lambda=\frac{1}{2}\left(\gamma_{1}+\gamma_{2}+\cdots+\gamma_{n}\right)
$$

is the highest weight for $T$ on $V_{\mathbb{C}}$. In particular, the restriction of $V$ to the subgroup $\mathrm{SL}_{2}(\mathbb{R})^{n}$ of $G$ given by the strongly orthogonal roots contains the irreducible representation $\left(\mathbb{R}^{2}\right)^{\otimes n}$, and the rank of $\mathcal{W}^{p, q}$ is $\geq\left(\begin{array}{l}n \\ p\end{array}\right)$.

Let $S^{\bullet}(V)=\bigoplus_{n \geq 0} S^{n}(V)$ be the symmetric algebra on $V$, and let $S^{\bullet}(V)^{G}$ denote the sub-algebra of $G$-invariants. We find [K, Tables II and III] that $V$ is a polar representation of $G$, and that $S^{\bullet}(V)^{G}$ is free, if and only if $n \leq 4$. More precisely:

$$
S^{\bullet}(V)^{G}=\left\{\begin{array}{lll}
\mathbb{R} & \text { if } & n=1 \\
\mathbb{R}\left[f_{2}\right] & \text { if } & n=2 \\
\mathbb{R}\left[f_{4}\right] & \text { if } & n=3 \\
\mathbb{R}\left[f_{2}, \cdots, f_{k}\right] & \text { if } & n=4
\end{array}\right.
$$

where $\operatorname{deg} f_{2}=2, \operatorname{deg} f_{4}=4$, and there are $\geq 4$ independent invariants $f_{d}$ of degrees $d=2, \cdots, k$ when $n=4$. For example, when $G=\mathrm{SL}_{2}(\mathbb{R})^{4}$ and $V=\left(\mathbb{R}^{2}\right)^{\otimes 4}$, the invariants are freely generated by polynomials $f_{2}, f_{4}, f_{4}^{\prime}, f_{6}$ of degrees $d=2,4,4,6$. For the three simple tube domains $D$ of rank 4 , the degrees of the generating invariants are given by the degrees of the generating invariants for the reflection representations of the Weyl groups of type $E$ :

$$
\left\{\begin{array}{llll}
G=S p_{8}(\mathbb{R}) & V=\Lambda_{0}^{4} \mathbb{R}^{8} & d=2,5,6,8,9,12 & W\left(E_{6}\right) \\
G=S U_{4,4} & V_{\mathbb{C}}=\Lambda^{4} \mathbb{C}^{8} & d=2,6,8,10,12,14,18 & W\left(E_{7}\right) \\
G=\operatorname{Spin}_{16}^{*} & V=\frac{1}{2} \text { spin } & d=2,8,12,14,18,20,24,30 & W\left(E_{8}\right)
\end{array}\right.
$$

7.

We return to the case when $D$ is simple. Let $\check{D}=G_{\mathbb{C}} / P_{\mathbb{C}}=\left(G_{1}\right)_{\mathbb{C}} /\left(P_{1}\right)_{\mathbb{C}}$ be the compact dual of $D$, and let $X=D^{ \pm} \hookrightarrow \check{D}$ be the Borel embedding [S, pp. 58-59]. Then $X$ is the unique open orbit of $G_{1}$ on $\check{D}$.

The equivariant vector bundles $\mathcal{W}^{p, q}$ on $X$ are all pull-backs of algebraic vector bundles on $\check{D}$. The line bundles $\omega=\mathcal{W}^{n, 0}$ and $\mathcal{L}=\mathcal{W}^{0, n}$ give the two generators of $\operatorname{Pic}(\check{D}) \simeq \mathbb{Z}$. The line bundle $\mathcal{L}$ is ample on $\check{D}$, and the canonical bundle of $\check{D}$ is isomorphic to $\omega^{2 d / n}$.

Let $\Sigma \subset \partial D$ be the Shilov boundary of $D$, which is the unique closed orbit $G / P$ of $G$ in $\check{D}$. An interesting question is to study the behavior of 
the variation $\mathcal{V}$ as one approaches a point $\sigma$ of the Shilov boundary $\Sigma$ of $X$. The resulting mixed Hodge structure $\mathcal{V}_{\sigma}$ "mirrors" that of $\mathcal{V}$. One has $\mathcal{W}_{\sigma}^{p, q}=0$ unless $p=q$, and the dimension of $\mathcal{W}_{\sigma}^{p, p}$ is equal to the dimension of $\mathcal{W}^{q, p}$, with $p+q=n$.

Let $\mathcal{A}^{q}=\mathcal{W}^{p, q} \otimes\left(\mathcal{W}^{n, 0}\right)^{-1}=\mathcal{W}^{p, q} \otimes \mathcal{L}$. The exact sequence $(5.3)$ of holomorphic, equivariant, algebra bundles

$$
0 \longrightarrow I \longrightarrow \operatorname{Sym}^{\bullet}(\Theta) \longrightarrow \bigoplus_{q=0}^{n} \mathcal{A}^{q} \longrightarrow 0
$$

on $X$ extends, as a sequence of complex algebraic, equivariant, algebra bundles, to $\check{D}=G_{\mathbb{C}} / P_{\mathbb{C}}$. It then descends to a sequence of algebra bundles over the real algebraic variety $\Sigma=G / P$, with complex points $\check{D}$. This gives an algebra structure $\bigoplus_{q=0}^{n} \mathcal{A}_{\mathbb{R}}^{q}$ on the limit mixed Hodge structure $\mathcal{V}_{\sigma}$ at points $\sigma$ of $\Sigma$, with ample cone $C \subset N=\left(\mathcal{A}_{\mathbb{R}}^{1}\right)_{\sigma}$.

\section{8.}

Another interesting question is whether the variation $\mathcal{V}$ occurs in nature (i.e. algebraic geometry). There one obtains local systems of $\mathbb{Q}$-vector spaces, so the first requirement is to specify a descent $\left(G_{\mathbb{Q}}, V_{\mathbb{Q}}\right)$ of the pair $(G, V)$ from $\mathbb{R}$ to $\mathbb{Q}$. In the cases $C_{n}$ ( $n$ odd) and $E_{7}, V$ is a faithful representation of $G$ and there is a unique descent of the pair $(G, V)$ to $\mathbb{Q}$. The resulting group $G_{\mathbb{Q}}$ is split over $\mathbb{Q}_{p}$ for all finite primes $p$. In the cases $C_{n}$ ( $n$ even), and in certain of the cases $B$ and $D^{\mathbb{R}}$, one can specify a descent by insisting that $G_{\mathbb{Q}}$ is split over $\mathbb{Q}_{p}$ for all finite primes $p$. In the other cases, a descent requires some choice — such as an imaginary quadratic field or a definite quaternion algebra over $\mathbb{Q}$.

Assume that a descent $\left(G_{\mathbb{Q}}, V_{\mathbb{Q}}\right)$ of the pair $(G, V)$ has been specified. One can then ask if there is a family $f: Y \rightarrow S$ of smooth complex polarized projective varieties, where the base $S=\Gamma \backslash D$ is uniformized by $D$ and $\Gamma$ is an arithmetic subgroup of $G_{\mathbb{Q}}$, such that $V_{\mathbb{Q}}$ is the pull-back to $D$ of the variation $\left(R^{n} f_{*} \mathbb{Q}\right)_{\text {primitive }}=V_{Y}$ on $S$. The limit mixed Hodge structure $\bigoplus_{q=0}^{n} \mathcal{A}_{\mathbb{Q}}^{q}$ will then be associated to degenerations in the family $f: Y \rightarrow S$ over the 0-dimensional cusps $\Gamma \backslash G_{\mathbb{Q}} / P_{\mathbb{Q}}$ of the Satake compactification $\bar{S}$.

\section{9.}

The simplest geometric families $f: Y \rightarrow S$ to study are those where the fibres $Y_{s}$ have dimension $n=\operatorname{rank}(D)$ and trivial canonical class $\left(c_{1}\left(Y_{s}\right)=\right.$ 0, or equivalently $\Omega_{Y_{s}}^{n} \simeq \mathcal{O}_{Y_{s}}$ ). Then $F^{n} \mathcal{V}_{Y}=f_{*} \Omega_{Y / S}^{n}$ is a holomorphic 
line bundle on $S$, and (when $S$ is a universal family) the Kodaira-Spencer map:

$$
\nabla: \Theta_{S} \longrightarrow \operatorname{Hom}\left(F^{n} \mathcal{V}_{Y}, F^{n-1} \mathcal{V}_{Y} / F^{n} \mathcal{V}_{Y}\right)
$$

is an isomorphism (cf. [Tn]). Hence, several of the key properties of $\mathcal{V}_{\mathbb{Q}}$ established in $\S 4$ hold for $\mathcal{V}_{Y}$.

For example, if one takes the descent $G_{\mathbb{Q}}=S p(2 n, \mathbb{Q})$ of $G=S p(2 n, \mathbb{R})$ to $\mathbb{Q}$, which is the only choice when $n$ is odd and is split at all finite primes $p$ in general, then $\mathcal{V}_{\mathbb{Q}}$ is the pull-back of $\mathcal{V}_{Y}$ for a universal family $f: Y \rightarrow S$ of polarized abelian varieties of dimension $=n$.

\section{0.}

More generally, $\mathcal{V}_{\mathbb{Q}}$ might arise from a sub-Hodge structure $\mathcal{V}_{Y, p} \subset \mathcal{V}_{Y}$ on $S$, where $p$ is a projector in the primitive cohomology of dimension $n$. For example, if $G=\operatorname{Spin}(2,10)$, the homogeneous cone $C$ associated to $D$ is isomorphic to $S_{2}(\mathbb{O})^{+}$, the cone of $2 \times 2$ positive definite symmetric matrices over the octonions. Since the octonions have a unique descent to $\mathbb{Q}$, one obtains a descent $G_{\mathbb{Q}}$ for $G$ which is split at all finite primes $p$. In this case $\mathcal{V}_{\mathbb{Q}}$ has type $(1,10,1)$ and arises as the pull-back of $\mathcal{V}_{Y}^{\sigma=-1}$, for a universal family $f: Y \rightarrow S$ of polarized K3 surfaces with an Enriques involution $\sigma$ (which is generically fixed point free on $Y_{s}$ ).

Can such a geometric realization be found in the case when $G=E_{7,3}$ and $C=S_{3}(\mathbb{O})^{+}$? Here there is a unique descent $\left(G_{\mathbb{Q}}, V_{\mathbb{Q}}\right)$ to $\mathbb{Q}$, and one is looking for a family of 3-folds $f: Y \rightarrow S$ with trivial canonical class, such that $\mathcal{V}_{\mathbb{Q}}$ is the pull-back of a sub-Hodge structure $\mathcal{V}_{Y, p} \subset \mathcal{V}_{Y}$.

The Hodge numbers of $\mathcal{V}_{\mathbb{Q}}$ are $(1,27,27,1)$. Hence we must have $h^{2,1}\left(Y_{s}\right) \geq$ 27; the general theory of 3-folds with $c_{1}=0$ then shows that one must have $h^{1,0}\left(Y_{s}\right)=h^{2,0}\left(Y_{s}\right)=0$. For reasons of mirror symmetry, it seems unlikely that $\mathcal{V}_{\mathbb{Q}}$ is the pull-back of the entire 3 -cohomology $\mathcal{V}_{Y}$. The simplest guess, in analogy with the case where $C=S_{2}(\mathbb{O})^{+}$, is that $\mathcal{V}_{\mathbb{Q}}$ arises from a subHodge structure $\mathcal{V}_{Y}^{\sigma=-1}$, where $\sigma$ is an involution which generically has 16 isolated fixed points on $Y_{s}$. Then $F^{3}\left(\mathcal{V}_{Y}^{\sigma=-1}\right)$ is a line bundle on $S$.

Any geometric realization $f: Y \rightarrow S$ of the variation $\mathcal{V}$ associated with $G=E_{7,3}$ must be fairly complicated, as Deligne has remarked that the associated Hodge structures have no Picard-Lefshetz degenerations. This excludes the many constructions of 3 -folds with $c_{1}=0$ which are given as smooth complete intersections in weighted projective spaces. Perhaps the existence of small resolutions of nodes in dimension 3 can be profitably used in this context. 


\section{References}

[D] P. Deligne, Variétés de Shimura: interprétation modulaire, et techniques de construction de modèles canoniques, Proc. Symp. Pure Math., Part 2, vol. 33, AMS, 1979, pp. 247-290.

[K] V. G. Kac, Some remarks on nilpotent orbits, J. Algebra 64 (1980), 190-213.

[M] J. S. Milne, Canonical models of (mixed) Shimura varieties and automorphic vector bundles, Perspectives in Math., vol. 10, Academic Press, 1990, pp. 83-414.

[S] I. Satake, Algebraic structures of symmetric domains., Princeton Univ. Press, 1980.

[Tn] G. Tian, Smoothness of the universal deformation space of compact Calabi-Yau manifolds and its Peterson-Weil metric, Math. Aspects of String Theory, World Scientific Press, 1987, pp. 629-646.

[T] J. Tits, Classification of algebraic semi-simple groups, Proc. Symp. Pure Math., vol. 9, AMS, 1966, pp. 33-62.

Department of Mathematics, Harvard University, Cambridge, M A 02138

E-mail address: gross@math.harvard.edu 